\title{
Decentralized Fault Detection and Fault-Tolerant Control for Nonlinear Interconnected Systems
}

\author{
Shun Zhou, Jianjun Bai and Feng $\mathrm{Wu}^{*}$ \\ Information and Control Institute, Hangzhou Dianzi University, Hangzhou 310018, China; \\ zhoushun0207@163.com (S.Z.); baijianjun@hdu.edu.cn (J.B.) \\ * Correspondence: fengwu@hdu.edu.cn; Tel.: +86-571-86915040
}

Citation: Zhou, S.; Bai, J.; Wu, F. Decentralized Fault Detection and Fault-Tolerant Control for Nonlinear Interconnected Systems. Processes 2021, 9, 591. https://doi.org/ $10.3390 /$ pr9040591

Academic Editor: Fernando V. Lima

Received: 7 February 2021

Accepted: 25 March 2021

Published: 29 March 2021

Publisher's Note: MDPI stays neutral with regard to jurisdictional claims in published maps and institutional affiliations.

Copyright: (c) 2021 by the authors. Licensee MDPI, Basel, Switzerland. This article is an open access article distributed under the terms and conditions of the Creative Commons Attribution (CC BY) license (https:// creativecommons.org/licenses/by/ $4.0 /)$.

\begin{abstract}
The nonlinear interconnected system is a complex and important system in daily production and life in general. Due to the interconnection influence between subsystems and external disturbance factors, the system is prone to failure. For this kind of system, a decentralized fault detection and fault tolerant control method is proposed here. Compared with the traditional control scheme, this paper designs a subsystem communication protocol to reduce the information exchange between subsystems. Based on this communication protocol, a fault detection scheme is then designed. Due to the existence of a fault detection threshold in this scheme, the system can detect the fault in time without missing it or having a false alarm. Under the assumed condition, the adaptive control rate is obtained by establishing the adaptive approximation model to approximate the upper bound of the fault, and the subsystem adaptively adjusts the control rate according to the fault condition, so that the system can quickly recover to stability. Finally, a simulation program is used to verify the proposed method.
\end{abstract}

Keywords: interconnection influence; decentralized; fault detection; fault-tolerant control; communication protocol

\section{Introduction}

In real life, interconnected systems are ubiquitous, such as power grid systems, transportation systems, aircraft hydraulic systems, injection molding systems, etc. An interconnected system usually refers to a complex high-dimensional control system with uncertainty, nonlinearity, dispersion, and coupling. Therefore, the modeling and stable control of interconnected systems is not easy and thus, the research on interconnected systems has real practical significance.

Because the interconnected system is huge and the connections between the various subsystems are intricate, the system is prone to failures due to internal interconnections and external disturbances during operation. Therefore, the use of decentralized control divides the interconnected system into several subsystems, and establishes a separate detector and controller for each subsystem, which reduces the connection between the subsystems. If each subsystem is stable, the interconnected system is also stable. In order to compensate for the adverse effects of a failure, the failure must be detected first, and then the system can be stabilized through fault-tolerant control.

There are two main methods of fault detection. The two detection schemes are described in detail in the literature [1]: data-driven and model-based. The data-driven method [2] requires not only the normal data of the system but also the error data, which requires a large amount of data transmission and calculation. In the model-based method [3], the system's mathematical model and sensor measurement values are mainly used to detect faults. The literature [4-7] are model-based analysis methods. Model-based schemes also utilize many different ways, such as using adaptive estimators [8,9], fuzzy observers [10], high-gain observers [11], neural network observers [12] and geometric techniques [13], as 
well as using the state estimator as a reference model for fault detection. On the other hand, centralized fault detection can also be realized by using observer-based schemes [14].

The traditional centralized fault detection has also been the subject of many literature studies. For example, literature [15] proposed a fault diagnosis method based on deep learning multi-model fusion. Long term memory (LSTM) and convolutional neural network $(\mathrm{CNN})$ are used to extract features. Then, the extracted features are fused and multilayer perceptron (MLP) is used as input for further feature compression extraction, and finally the diagnosis results are obtained. In reference [16], an adaptive iterative learning algorithm based on the Runge-Kutta fault estimation observer model was proposed to solve the problems of large estimation error and slow convergence rate in the process of fault detection and estimation of nonlinear systems, which effectively reduced the fault estimation error.

The above-mentioned centralized fault detection scheme is suitable for small or low order systems, but it is not suitable for large interconnected systems such as power generation systems and transportation systems, because a large amount of data transmission and processing is required between the subsystems of interconnected systems. In addition, for such a large-scale interconnected system, the centralized solution is costly and time-consuming. Therefore, literature [17] proposed a fault diagnosis scheme combining decentralization and distribution.

In the decentralized fault detection scheme [18,19], the large continuous time system is decomposed into several subsystems, among which the subsystems are connected by nonlinear functions. However, these papers assume that the interconnection functions are known and that the estimated vectors of the system states are available, which is a shortcoming. Therefore, in this paper a decentralized fault detection scheme is designed, assuming the interconnection function between subsystems and the external disturbance function are unknown. Based on the communication protocol between the subsystems, a state estimation model is designed for each subsystem according to the state of the subsystem, when the norm of this state error exceeds the set threshold, a fault is detected.

When the fault is detected, the next step is to conduct fault-tolerant control on the failed subsystem. Fault-tolerant control means that when the system fails, it can operate within the acceptable performance range through the system's self-adjustment [20,21].

Fault-tolerant control methods can be divided into two categories: hardware redundancy and analytical redundancy. Hardware redundancy is to achieve fault tolerance through redundancy backup of important components and components that are prone to failure; analytical redundancy is to adjust the control parameters or control the structure after a fault occurs to offset the dynamic changes caused by the fault. This article uses a fault-tolerant control method based on analytical redundancy.

The problem of decentralized fault-tolerant control of interconnected systems is how to solve the interconnection effect between subsystems when the interconnection effect is unknown. Literature [22] designed an adaptive decentralized tracking control strategy based on the backstepping design method and the approximate characteristics of neural network respectively for known and unknown quantized parameters. Literature [23] proposes a decentralized neural network controller for approximating unknown subsystems and interconnection functions. For interconnected nonlinear systems, literature [24] designed an iterative learning control law based on P-type learning law, so that the controller of each subsystem only depends on the output variables of the subsystem and does not need to exchange information with other subsystems. These control schemes regard the interconnection function and the external disturbance function as a whole, and approach the sum of them by neural network, but the interconnection function and the external disturbance function are not necessarily the same type. If the two are regarded as a whole, the neural network scheme can not effectively approximate the sum of them, resulting in the low stability of the system. Therefore, the decentralized fault-tolerant control rate is divided into two parts in this paper. One part is used to Therefore, the decentralized fault-tolerant control rate is divided into two parts in this paper. One part is used to make 
up for the influence of the interconnection effect, and the other part is used to solve the dynamic change caused by external disturbance and accurately control it. Through this control method, the system can quickly recover to a stable state after a failure.

\section{Subsystem Model}

Consider a system consisting of $n$ interconnected subsystems. The system may have multiple external disturbances at unknown times. The $i$-th $(i=1,2 \ldots n)$ subsystem model is:

$$
\begin{aligned}
& \dot{x}_{i j}=x_{i(j+1)} \quad(j=1,2 \ldots, m-1) \\
& \dot{x}_{i m}=f_{i}\left(x_{i}\right)+g_{i}\left(x_{i}\right) u_{i}+\sum_{j=1}^{n} \varphi_{i j}\left(x_{j}\right)+\sum_{a=1}^{b_{i}} \Omega\left(t-T_{i a}\right) h_{i a}\left(x, u_{i}\right) \\
& y_{i}=C_{i} x_{i}
\end{aligned}
$$

Write the subsystem in matrix form as:

$$
\begin{aligned}
\dot{x}_{i} & =A_{i} x_{i}+B_{i}\left[f_{i}\left(x_{i}\right)+g_{i}\left(x_{i}\right) u_{i}+\sum_{j=1}^{n} \varphi_{i j}\left(x_{j}\right)+\sum_{a=1}^{b_{i}} \Omega\left(t-T_{i a}\right) h_{i a}\left(x, u_{i}\right)\right] \\
y_{i} & =C_{i} x_{i}
\end{aligned}
$$

where

$$
A_{i}=\left[\begin{array}{ccccc}
0 & 1 & 0 & \cdots & 0 \\
0 & 0 & 1 & \cdots & 0 \\
\vdots & \vdots & \vdots & \ddots & \vdots \\
0 & 0 & 0 & \cdots & 1 \\
0 & 0 & 0 & \cdots & 0
\end{array}\right]_{m \times m} \quad B_{i}=\left[\begin{array}{c}
0 \\
0 \\
\vdots \\
0 \\
1
\end{array}\right]_{m \times 1} \quad C_{i}=[1,0, \ldots 0]_{1 \times m}
$$

where $x_{i}=\left[x_{i 1}, x_{i 2}, \ldots \ldots x_{i m}\right]^{T} \in \Re^{n_{i}}$ represents the state vector of the $i$-th subsystem, $x=\left[x_{1}, x_{2} \ldots \ldots x_{n}\right] \in \Re^{n}$ represents the state of the entire system, and $u_{i}$ represents the control input of the $i$-th subsystem, where $f_{i}\left(x_{i}\right): \Re^{m_{i}} \rightarrow \Re$ and $g_{i}\left(x_{i}\right): \Re^{m_{i}} \rightarrow \Re$ are known functions and represent the local dynamics of the subsystem. Different from literature $[25,26]$, the function $f_{i}, g_{i}$ of subsystem $\mathrm{i}$ in this paper can be a function of all state variables $\left(x_{i 1}, x_{i 2}, \ldots \ldots x_{i m}\right)$ of the $i$-th subsystem. $\varphi_{i j}$ is an unknown function, representing the interconnection effect between the $i$-th subsystem and the $j$-th subsystem, and $\varphi_{i i}=0 . h_{i a}(x)$ represents the ath external disturbance function of subsystem $i$. This paper considers that there may be multiple disturbances in each subsystem, where $b_{i}$ represents the total number of disturbances in the $i$-th subsystem. If there is no external disturbance in subsystem $i$, then $b_{i}=0 . \Omega\left(t-T_{i a}\right)$ represents the disturbance coefficient distribution corresponding to the disturbance occurring at the unknown time $T_{i a}$.

$$
\Omega\left(t-T_{i a}\right)= \begin{cases}0 & \text { if } t<T_{i a} \\ 1-e^{-D_{i}\left(t-T_{i a}\right)} & \text { if } t \geq T_{i a}\end{cases}
$$

where $D_{i}>0$ is an unknown constant representing the rate of disturbance. The large $D_{i}$ value indicates sudden disturbance, and the relatively small $D_{i}$ value indicates a disturbance that occurs slowly. In order to ensure the controllability of the scheme, assume that each input gains function $g_{i}\left(x_{i}\right)>0$.

The block diagram of the subsystem state equation is shown in Figure 1: 


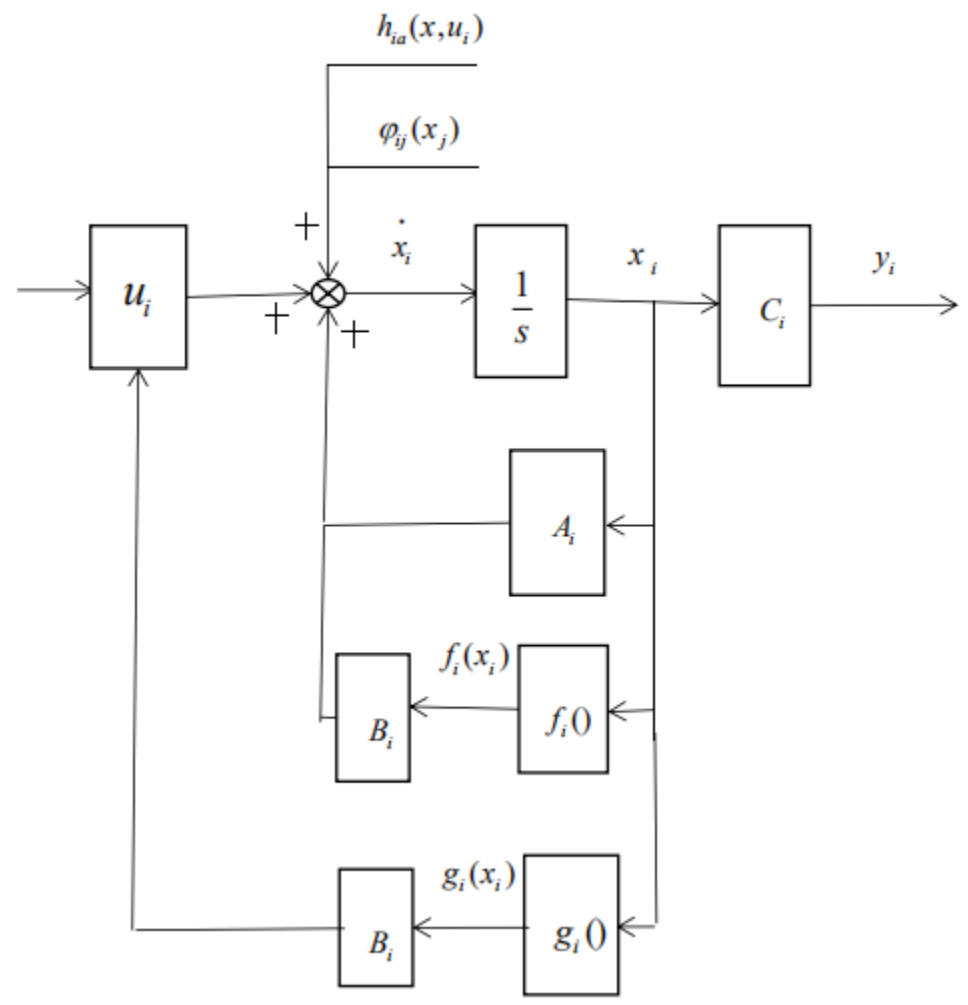

Figure 1. Block diagram of the state equation of the subsystem.

In Figure $1, f_{i}()$ and $g_{i}()$ represent nonlinear functions of $x_{i}$. Suppose the local nominal model of the $i$-th subsystem is:

$$
\dot{x}_{N_{i}}=A_{i} x_{N_{i}}+B_{i}\left[f_{N_{i}}\left(x_{N_{i}}\right)+g_{N_{i}}\left(x_{N_{i}}\right) u_{i}\right]
$$

where

$$
\begin{aligned}
& \left|f_{i}\left(x_{i}\right)-f_{N_{i}}\left(x_{i}\right)\right| \leq f_{0_{i}}\left(x_{i}\right), \forall x_{i} \in \Re^{n_{i}} \\
& \left|g_{i}\left(x_{i}\right)-g_{N_{i}}\left(x_{i}\right)\right| \leq g_{0_{i}}\left(x_{i}\right), \forall x_{i} \in \Re^{n_{i}}
\end{aligned}
$$

$f_{0_{i}}\left(x_{i}\right)$ and $g_{0_{i}}\left(x_{i}\right)$ are known local boundary functions, representing the boundaries of the modeling uncertainty of $f_{i}$ and $g_{i}$, respectively. In order to avoid any stability issues and without loss of generality, assume that $g_{N_{i}}\left(x_{i}\right)-g_{0_{i}}\left(x_{i}\right)$ is far from zero and positive for all $x_{i} \in \Re^{n_{i}}$. To ensure that $g_{i}\left(x_{i}\right)>0$ for all $x_{i} \in \Re^{n_{i}}$, it is assumed that the interconnection function $\varphi_{i j}$ is bounded.

Assumption 1. The higher-order term after Taylor expansion of $\varphi_{i j}$ satisfies the Equation (5) for all $i \neq j$.

$$
\left|\varphi_{i j}\left(x_{j}\right)\right| \leq L_{i j}\left|x_{j}\right|+\sigma_{j}, \forall x_{j} \in \Re^{n_{j}}
$$

where $L_{i j}$ and $\sigma_{j}$ are known constants, and $\left|x_{j}\right|=\sqrt{x_{j 1}^{2}+\ldots+x_{j m}^{2}}$ is the Euclidean norm. The goal of this paper was to develop a fault detection and fault-tolerant control scheme for the nonlinear interconnection subsystem described by (1), so that each $x_{i}$ follows a smooth reference trajectory vector $x_{d_{i}}=\left[x_{d_{i 1}}, x_{d_{i 1}}, \ldots, x_{d_{i n}}\right]^{T}$.

\section{Subsystem Communication Protocol}

Suppose $x_{d_{i}}$ is the expected state vector of the $i$-th subsystem, $x_{d_{i}}$ and $\dot{x}_{d_{i}}$ are bounded and hold for all subsystems. Let $\widetilde{x}_{i j}=x_{i j}-x_{d_{i j}}$ be the tracking error of the $j$-th state of the $i$-th subsystem. The tracking error vector of the $i$-th subsystem is defined as $\widetilde{x}_{i}=\left[\widetilde{x}_{i 1}, \widetilde{x}_{i 2}, \ldots \widetilde{x}_{i m}\right]^{T}$. 
Figure 2 shows the subsystem communication protocol. The communication between subsystems is mainly through the interconnection function. The interconnection function is a function composed of the state vectors of the interconnected subsystems. In order to reduce the communication between the subsystems, the communication threshold is set in this paper. Specifically, when the norm of tracking error $\widetilde{x}_{i}(t)$ of the $i$-th subsystem exceeds a certain threshold value $d_{i}$ (where $d_{i}$ is a design constant), its actual state $x_{i}(t)$ is provided to other subsystems connected to it through the interconnection function, otherwise, the interconnection function of other subsystems will use the existing known desired state $x_{d_{i}}$.

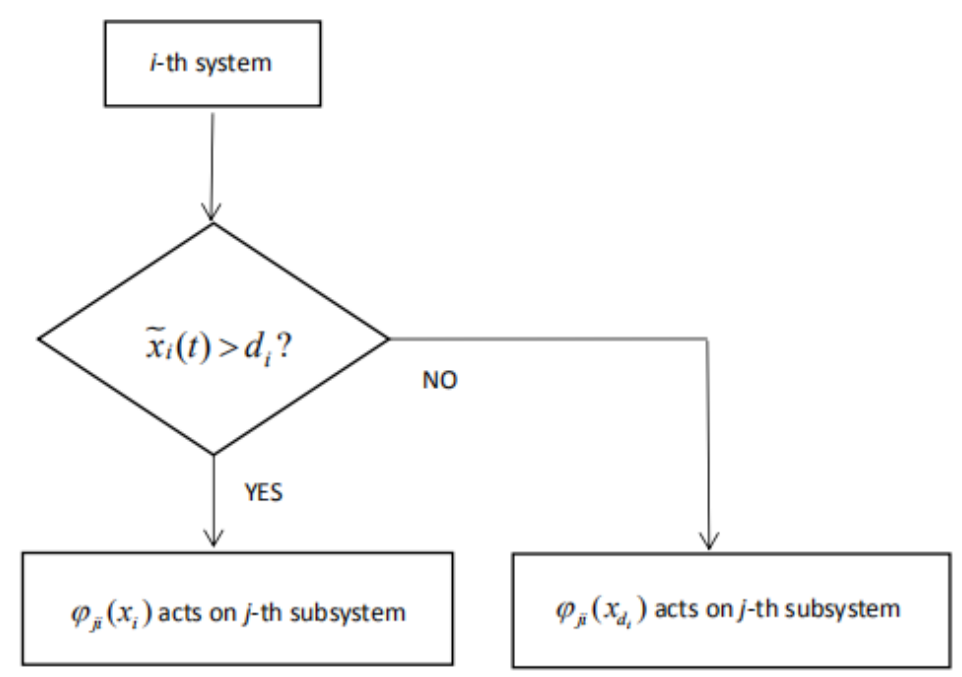

Figure 2. Subsystem communication protocol.

Suppose $t_{i k}^{a}$ is the time when the $i$-th subsystem starts to transmit its state to other subsystems at the kth time, $t_{i k}^{b}$ is the time when the k-th time stops transmitting its state to other subsystems, and $t_{i k}^{a}<t_{i k}^{b}$. Define the vector $\bar{x}_{i}=\left[\bar{x}_{1}, \bar{x}_{2}, \ldots, \bar{x}_{n}\right]^{T}$, where $\bar{x}_{i}$ takes the following value:

$$
\bar{x}_{i}=\left\{\begin{array}{l}
x_{i}, t \in\left[t_{i k}^{a}, t_{i k}^{b}\right) \\
x_{d_{i}}, t \in\left[t_{i k}^{b}, t_{i(k+1)}^{a}\right)
\end{array}\right.
$$

In many large-scale interconnected systems, it is necessary to reduce the communication within the system while maintaining high performance. Therefore, this paper designs a communication protocol to reduce the information exchange between subsystems. That is, when the norm of the tracking error $\widetilde{x}_{i}(t)$ is higher than a certain constant value $d_{i}$, the subsystem state $x_{i}(t)$ will be communicated to other subsystems through the interconnection function. Therefore, when $t \in\left[t_{i k}^{a}, t_{i k}^{b}\right)$, the components of the interconnection function of other subsystems connected to it adopt their actual state, assuming that all subsystems can use the required reference trajectory $x_{d_{i}}(i=1,2 \ldots n)$ to ensure that

$$
G\left(x_{i}\right)=\left\{\begin{array}{l}
\left|x_{i}-\bar{x}_{i}\right|=0, t \in\left[t_{i k}^{a}, t_{i k}^{b}\right) \\
\left|x_{i}-\bar{x}_{i}\right| \leq d_{i}, t \in\left[t_{i k}^{b}, t_{i(k+1)}^{a}\right)
\end{array}\right.
$$

\section{Decentralized Fault Detection Scheme}

In this section, the decentralized fault detection scheme of the nonlinear estimator is designed for each subsystem. The estimator of the $i$-th subsystem is:

$$
\hat{\hat{x}}_{i}=\lambda_{i}\left(x_{i}-\hat{x}_{i}\right)+A_{i} \hat{x}_{i}+B_{i}\left[f_{N_{i}}\left(x_{i}\right)+g_{N_{i}}\left(x_{i}\right) u_{i}+\operatorname{sgn}\left(E\left(x_{i}\right)\right) \sum_{j=1}^{n}\left(L_{i j}\left|\bar{x}_{j}\right|+\sigma_{j}\right)\right]
$$




$$
E\left(x_{i}\right)=\left\{\begin{array}{c}
1, t \in\left[t_{i k}^{a}, t_{i k}^{b}\right) \\
0, t \in\left[t_{i k}^{b}, t_{i(k+1)}^{a}\right)
\end{array}\right.
$$

where $\hat{x}_{i} \in \Re$ is the estimated state of the $i$-th subsystem. In order to avoid the influence of modeling uncertainty and noise and other factors on the system model, $f_{N_{i}}\left(x_{i}\right)$ and $g_{N_{i}}\left(x_{i}\right)$ in nominal model Equation (2) are used to replace $f_{i}\left(x_{i}\right)$ and $g_{i}\left(x_{i}\right)$ in Equation (1) in the estimator model Equation (7). Satisfying $\hat{x}_{i}(0)=x_{i}(0) . \lambda_{i}>0$ is a custom scalar [27], which can better track the state vector $x_{i}$ by adjusting the size of $\lambda_{i}$. Based on the communication protocol between subsystems, when the interconnection between subsystems occurs, $\operatorname{sgn}\left(E\left(x_{i}\right)\right)=1$, and $\left(\sum_{j=1}^{n} L_{i j}\left|\bar{x}_{j}\right|+\sigma_{j}\right)$ is introduced into the estimator model Equation (7); when there is no interconnection between subsystems, $\operatorname{sgn}\left(E\left(x_{i}\right)\right)=0$, and the estimator model does not need to introduce the interconnection function. The estimator model in literature [28] always contains an interconnection function. Although the value of the interconnection function is small, this paper only introduces an interconnection function when communication occurs between subsystems, so as to make the size of the estimator $\hat{x}_{i}$ more accurate. Let the estimation error $\varepsilon_{i}=x_{i}-\hat{x}_{i}$ be used for fault detection.

For each subsystem, we define the detection threshold $R_{i}(t)$, as shown in Equation (8).

$$
R_{i}(t)=\int_{0}^{t} e^{\left(A_{i}-\lambda_{i} E\right)(t-\tau)} B\left[f_{0_{i}}\left(x_{i}(\tau)\right)+g_{0_{i}}\left(x_{i}(\tau)\right)\left|u_{i}\right|+\sum_{j=1}^{n} L_{i j} r_{j}\right] d \tau
$$

where

$$
r_{j}=\left\{\begin{array}{l}
0, t \in\left[t_{j k}^{a}, t_{j k}^{b}\right) \\
d_{j}, t \in\left[t_{j k}^{b}, t_{j(k+1)}^{a}\right.
\end{array}\right)
$$

If $\left|\varepsilon_{i}\left(t_{d}^{i}\right)\right| \geq R_{i}\left(t_{d}^{i}\right)$, the fault alarm occurs at time $t_{d}^{i}$. The theorem 1 shows that in the absence of any fault, the state estimation error satisfies $\left|\varepsilon_{i}(t)\right|<R_{i}(t)$, so it can ensure that there is no false alarm.

Theorem 1. If the fault detection estimator described in Equation (7) satisfies Equation (10):

$$
\left|\varepsilon_{i}(t)\right| \leq R_{i}(t) \forall t \in T_{i}
$$

Then the decentralized fault detection estimator given by Equation (7) guarantees that as long as Equation (10) is not satisfied, the i-th subsystem will fail.

Proof. Based on Equations (2) and (7), the state estimation error of the $i$-th subsystem dynamically satisfies:

$$
\dot{\varepsilon}_{i}=\left(A_{i}-\lambda_{i} E\right) \varepsilon_{i}+B_{i}\left[f_{i}\left(x_{i}\right)+g_{i}\left(x_{i}\right) u_{i}+\sum_{i=i}^{n} \varphi_{i j}\left(x_{j}\right)+\sum_{a=1}^{b_{i}} \Omega\left(t-T_{i a}\right) h_{i a}(x)-f_{N_{i}}\left(x_{i}\right)-g_{N_{i}}\left(x_{i}\right) u_{i}-\left(\sum_{j=1}^{n} L_{i j}\left|\bar{x}_{j}\right|+\sigma_{j}\right)\right]
$$

In the case of no failure, $t<T_{i a}$, the state estimation error of the $i$-th subsystem dynamically satisfies:

$$
\dot{\varepsilon}_{i}=\left(A_{i}-\lambda_{i} E\right) \varepsilon_{i}+B_{i}\left[f_{i}\left(x_{i}\right)+g_{i}\left(x_{i}\right) u_{i}+\sum_{i=1}^{n} \varphi_{i j}\left(x_{j}\right)-f_{N_{i}}(x)-g_{N_{i}}\left(x_{i}\right) u_{i}-\left(\sum_{j=1}^{n} L_{i j}\left|\bar{x}_{j}\right|+\sigma_{j}\right)\right]
$$

By integrating $\dot{\varepsilon}_{i}$, we can get

$$
\varepsilon_{i}(t)=\int_{0}^{t} e^{\left(A_{i}-\lambda_{i} E\right)(t-\tau)} B_{i}\left[f_{i}\left(x_{i}(\tau)\right)+g_{i}\left(x_{i}(\tau)\right) u_{i}+\sum_{i=1}^{n} \varphi_{i j}(x(\tau))-f_{N_{i}}(x(\tau))-g_{N_{i}}\left(x_{i}(\tau)\right) u_{i}-\left(\sum_{j=1}^{m} L_{i j}\left|\bar{x}_{j}(\tau)\right|+\sigma_{j}\right)\right] d \tau
$$


This can be obtained through Equations (3)-(5)

$$
\left|\varepsilon_{i}(t)\right| \leq \int_{0}^{t} e^{\left(A_{i}-\lambda_{i} E\right)(t-\tau)} B_{i}\left[f_{0_{i}}\left(x_{i}(\tau)\right)+g_{0_{i}}\left(x_{i}(\tau)\right)\left|u_{i}\right|+\sum_{j=1}^{n}\left(\left|L_{i j}\right|\left|x_{j}(\tau)\right|\left|\sigma_{j}\right|-L_{i j}\left|\bar{x}_{j}(\tau)\right|-\left|\sigma_{j}\right|\right)\right] d \tau
$$

Use Equation (6) to get

$$
\left|\varepsilon_{i}(t)\right| \leq \int_{0}^{t} e^{\left(A_{i}-\lambda_{i} E\right)(t-\tau)} B_{i}\left[f_{0_{i}}\left(x_{i}(\tau)\right)+g_{0_{i}}\left(x_{i}(\tau)\right)\left|u_{i}\right|+\sum_{j=1}^{n} L_{i j} r_{j}\right] d \tau=R_{i}(t)
$$

Therefore, the decentralized fault estimator given by Equation (7) guarantees that the $i$-th subsystem will fail whenever Equation (10) is not established.

Since there is a fault detection threshold in the fault detection scheme in this paper, even if there is a communication delay, as long as the state estimation difference exceeds the threshold, the system is considered to have a fault. Then, the system is adjusted through the fault-tolerant control scheme in the next section to ensure that the system can quickly restore stability after failure.

\section{Decentralized Fault-Tolerant Control Design}

Based on Equation (1), the dynamic equation $\tilde{x}_{i}=x_{i}-x_{d_{i}}$ of the tracking error of the $i$-th subsystem satisfies:

$$
\begin{aligned}
& \dot{\tilde{x}}_{i j}=\tilde{x}_{i(j+1)} \quad(j=1,2, \ldots, m-1) \\
& \dot{\tilde{x}}_{i m}=f_{i}\left(x_{i}\right)+g_{i}\left(x_{i}\right) u_{i}+\sum_{j=1}^{n} \varphi_{i j}\left(x_{j}\right)+\sum_{a=1}^{b_{i}} \Omega\left(t-T_{i a}\right) h_{i a}\left(x, u_{i}\right)-\dot{x}_{d_{i m}}
\end{aligned}
$$

The dynamic equation of the tracking error can be written in the matrix form shown in Equation (12).

$$
\dot{\tilde{x}}_{i}=A_{i} \widetilde{x}_{i}+B_{i}\left[f_{i}\left(x_{i}\right)+g_{i}\left(x_{i}\right) u_{i}+\sum_{j=1}^{n} \varphi_{i j}\left(x_{j}\right)+\sum_{a=1}^{b_{i}} \Omega\left(t-T_{i a}\right) h_{i a}\left(x, u_{i}\right)-\dot{x}_{d_{i m}}\right]
$$

Let the control law based on decentralized adaptive control be:

$$
u_{i}=u_{i 1}+u_{i 2}
$$

where, $u_{i 1}$ is the control rate that stabilizes the subsystem under the condition of no external disturbance $\left(h_{i a}=0\right.$ ) but only the interconnection effect, while $u_{i 2}$ is the control rate of augmented fault regulation, which is used to solve the dynamic changes caused by the external disturbance. In reference [29], $u_{i 1}$ is the nominal control rate, and $u_{i 2}$ is used to compensate the influence of the faults. By adding the interconnection function and the external disturbance function, the control rate $u_{i 2}$ is adjusted by approaching the upper limit of the sum of the two. Since Assumption 1 in this paper has given the boundary of the interconnection function, $u_{i 1}$ approximates the upper limit of the interconnection function by Assumption 1 to compensate for the influence of the interconnection function within the subsystem. Then $u_{i 2}$ approaches the upper limit of the external perturbation function adaptively to compensate for the influence of the external perturbation function. The internal interconnection and external disturbance are controlled separately, which reduces the computation of $u_{i 2}$.

The control law $u_{i 1}$ of the ith subsystem is defined as Equation (13).

$$
u_{i 1}=\frac{-K_{i}^{T} \widetilde{x}_{i}-f_{i}\left(x_{i}\right)+\dot{x}_{d_{i m}}}{g_{i}\left(x_{i}\right)}-\frac{\operatorname{sgn}\left(E\left(x_{i}\right)\right)\left(\sum_{j=1}^{n} L_{i j}\left|\bar{x}_{j}\right|+\sigma_{j}\right)}{g_{i}\left(x_{i}\right)}
$$


Select the vector $K_{i}=\left[k_{i 1}, k_{i 2}, \ldots k_{i m}\right]^{T} \in \Re^{m_{i}}$ such that $A-B K_{i}^{T}$ is the Hurwitz matrix. Since the matrix $A-B K_{i}^{T}$ satisfies Hurwitz, for any $Q_{i}>0$, there exists a matrix $P_{i}$ that satisfies the Lyapunov equation such that $P_{i}\left(A-B K_{i}^{T}\right)+\left(A-B K_{i}^{T}\right)^{T} P_{i}=-Q_{i}$, define the scalar tracking error $e_{i}=B^{T} P_{i} \widetilde{x}_{i}$, and the external disturbance function $h_{i a}$ satisfies the Assumption 2.

Assumption 2. The external disturbance function $h_{i a}$ is bounded, and there is an unknown analytic function $\gamma_{i j}$ that makes the Equation (14) true.

$$
\sum_{a=1}^{b_{i}} \Omega\left(t-T_{i a}\right)\left|h_{i a}\left(x, u_{i}\right)\right| \leq \sum_{j=1}^{n} \gamma_{i j}\left(\left|e_{j}\right|\right)
$$

According to Equation (14), the fault-tolerant problem of the decentralized control scheme is simplified in solving the unknown boundary function $\gamma_{i j}$. This paper uses an adaptive approximation model and a linear parameterized approximator $s_{i}\left(e_{i}\right)$ to adaptively approximate the boundary function $\gamma_{i j}$ for use in the control law.

$$
s_{i}\left(e_{i}\right)=\phi_{s_{i}}\left(e_{i}\right)^{T} \theta_{s_{i}}+\mu_{s_{i}}\left(e_{i}\right)
$$

where $\phi_{s_{i}}$ is a set of basis functions, $\theta_{s_{i}}$ is a set of unknown constant parameters, and $\mu_{s_{i}}\left(e_{i}\right)$ is the residual approximation error. Generally, the residual approximation error is small in a certain area, but may become larger outside the area. Since $s_{i}\left(e_{i}\right)$ is by definition a substitute for the boundary function $\gamma_{i j}$, by choosing a given approximation model $\phi_{s_{i}}\left(e_{i}\right)^{T} \theta_{s_{i}}, s_{i}\left(e_{i}\right)$ can be approximated more accurately and smoothly, so that the residual approximation error can be as small as possible in this area. However, even a small approximation error will cause instability problems to the feedback control scheme due to parameter drift. In order to solve this problem, the dead zone correction can be combined with the adaptive boundary method in the adaptive control law. Assume that the approximation of the unknown function $s_{i}\left(e_{i}\right)$ given by Equation (12) is valid for a certain compact set. In this tight set, the residual approximate error $\mu_{s_{i}}\left(e_{i}\right)$ has an upper bound $\bar{\mu}_{s_{i}}$ namely $\left|\mu_{s_{i}}\left(e_{i}\right)\right|<\bar{\mu}_{s_{i}}$. The unknown $\bar{\mu}_{s_{i}}$ is estimated online, and adaptive estimation is used, which is denoted as $\hat{\mu}_{s_{i}}$. The augmented control rate $u_{i 2}$ of the $i$-th subsystem is defined as the Equation (16):

$$
\begin{gathered}
u_{i 2}=\frac{\operatorname{sgn}\left(\left|e_{i}\right|\right)\left(-\phi_{s_{i}}\left(e_{i}\right)^{T} \hat{\theta}_{s_{i}}-u_{m_{i}}\right)}{g_{i}\left(x_{i}\right)} \\
u_{m_{i}}=\left\{\begin{array}{cc}
\operatorname{sgn}\left(e_{i}\right) \hat{\mu}_{s_{i}} & , \widetilde{x}_{i}^{T} P_{i} \widetilde{x}_{i}>\lambda_{P_{i}} \varsigma_{i}^{2} \\
0 & , \widetilde{x}_{i}^{T} P_{i} \widetilde{x}_{i} \leq \lambda_{P_{i}} \varsigma_{i}^{2}
\end{array}\right.
\end{gathered}
$$

where $\varsigma_{i}>0$ is the design constant and $\lambda_{P_{i}}$ is the maximum eigenvalue of $P_{i}$. The parameter estimates of the adaptive approximator $\hat{\theta}_{s_{i}}$ and the adaptive boundary parameter $\hat{\mu}_{s_{i}}$ are updated according to the adaptive low of Equation (18):

$$
\begin{gathered}
\dot{\hat{\theta}}_{s_{i}}=\Gamma_{s_{i}} \phi_{s_{i}}\left(e_{i}\right) q_{i}\left(e_{i}, \tilde{x}_{i}, s_{i}\right) \\
\dot{\hat{\mu}}_{s_{i}}=\gamma_{s_{i}}\left|q_{i}\left(e_{i}, \tilde{x}_{i}, \varsigma_{i}\right)\right|
\end{gathered}
$$

where $\Gamma_{s_{i}}$ is a positive definite matrix, $\gamma_{s_{i}}$ is a normal number, which represents the adaptive gain of parameter estimation, and $q_{i}\left(e_{i}, \tilde{x}_{i}, s_{i}\right)$ is a dead zone, which is defined as Equation (20):

$$
q_{i}\left(e_{i}, \widetilde{x}_{i}, \varsigma_{i}\right)= \begin{cases}e_{i} & , \widetilde{x}_{i}^{T} P_{i} \widetilde{x}_{i}>\lambda_{P_{i}} \varsigma_{i}^{2} \\ 0 & , \widetilde{x}_{i}^{T} P_{i} \widetilde{x}_{i} \leq \lambda_{P_{i}} \varsigma_{i}^{2}\end{cases}
$$


The overall decentralized control law of the $i$-th subsystem is given by Equation (21):

$$
u_{i}=\frac{-K_{i}^{T} \widetilde{x}_{i}-f_{i}\left(x_{i}\right)-\phi_{s_{i}}\left(e_{i}\right)^{T} \hat{\theta}_{s_{i}}-u_{m_{i}}+\dot{x}_{d_{i m}}}{g_{i}\left(x_{i}\right)}-\frac{\operatorname{sgn}\left(E\left(x_{i}\right)\right)\left(\sum_{j=1}^{n} L_{i j}\left|\bar{x}_{j}\right|+\sigma_{j}\right)}{g_{i}\left(x_{i}\right)}
$$

The decentralized control law of the $i$-th subsystem given by (16) uses an adaptive approximation model to establish fault tolerance for all faults that meet (14). Generally, in a control law based on adaptive approximation, the unknown functions are compensated by adaptively approximating these unknown functions. However, in this paper, in order to ensure the dispersibility of the control scheme, the unknown interconnection effect and disturbance function can be compensated by adaptively approximating the substitute value of the boundary function $\gamma_{i j}$.

When the adaptive boundary term $\hat{\mu}_{s_{i}}$ is introduced, any small $\varsigma_{i}$ can be used, where $\varsigma_{i}$ represents the width of the dead zone. In addition, it avoids the need for a known upper limit of the residual approximation error $\bar{\mu}_{s_{i}}$. Although the non-decreasing increase of $\hat{\mu}_{s_{i}}$ may cause a larger feedback signal, it can only increase within a limited time interval due to the existence of the dead zone.

In this paper, we study two kinds of faults, one is caused by the interconnection function between systems, the other is caused by external disturbance. When there are multiple connecting units, the influence of noise can be included in the external perturbation function. This is because the adaptive approximation model established in this paper can approximate the upper bound of the external perturbation function (including noise) to the maximum extent, and then the size of $u_{i 2}$ can be adjusted adaptively to ensure the stable operation of the system.

\section{Stability Analysis}

Although the interconnection function $\varphi_{i j}\left(x_{j}\right)$ and the disturbance function $h_{i a}(x)$ are not only the function of the local state $x_{i}$, but also the function of the state of the subsystem $x_{j}, j \neq i$, the following proof shows that the output of each subsystem can be tracked asymptotically within a small error reference signals without exchanging status information between subsystems.

Lemma 1. For the interconnected system (1), the closed-loop system described by the decentralized control law (21) and the adaptive law (18), (19) ensures that $\left\|\widetilde{x}_{i}(t)\right\|$ is ultimately bounded by $\varsigma_{i}$, that is, the total time of $\widetilde{x}_{i}^{T} P_{i} \widetilde{x}_{i}>\lambda_{P_{i}} s_{i}^{2}$ is limited.

Proof. The Lyapunov function of the $i$-th subsystem is given by $V_{i}=V_{i 1}+V_{i 2}$, where

$$
\begin{aligned}
& V_{i 1}=\frac{1}{2} \widetilde{x}_{i}^{\top} P_{i} \widetilde{x}_{i} \\
& V_{i 2}=\frac{1}{2} \widetilde{\theta}_{s i}^{\top} \Gamma_{s i}^{-1} \widetilde{\theta}_{s_{i}}+\frac{1}{2 \gamma_{s_{i}}}\left(\hat{\mu}_{s_{i}}-\bar{\mu}_{s_{i}}\right)^{2}
\end{aligned}
$$

where $\tilde{\theta}_{s_{i}}=\hat{\theta}_{s_{i}}-\theta_{s_{i}}$ is the parameter estimation error vector. Substitute the control law (21) into the tracking error dynamic (12) to obtain the tracking error dynamic expression:

$$
\dot{\widetilde{x}}_{i}=\left(A_{i}-B_{i} K_{i}^{T}\right) \widetilde{x}_{i}+B_{i}\left(\sum_{j=1}^{n} \varphi_{i j}\left(x_{j}\right)+\sum_{a=1}^{b_{i}} \Omega\left(t-T_{i a}\right) h_{i a}\left(x, u_{i}\right)-\phi_{s_{i}}\left(e_{i}\right)^{T} \hat{\theta}_{s_{i}}-\operatorname{sgn}\left(E\left(x_{i}\right)\right)\left(\sum_{j=1}^{n} L_{i j}\left|\bar{x}_{j}\right|+\sigma_{j}\right)-u_{m_{i}}\right)
$$

The time derivative of $V_{i 1}$ satisfies:

$$
\dot{V}_{i 1}=-\frac{1}{2} \widetilde{x}_{i}^{T} Q_{i} \widetilde{x}_{i}+e_{i}\left(\sum_{j=1}^{n} \varphi_{i j}\left(x_{j}\right)+\sum_{a=1}^{b_{i}} \Omega\left(t-T_{i a}\right) h_{i a}\left(x, u_{i}\right)-\phi_{s_{i}}\left(e_{i}\right)^{T} \hat{\theta}_{s_{i}}-\operatorname{sgn}\left(E\left(x_{i}\right)\right)\left(\sum_{j=1}^{n} L_{i j}\left|\bar{x}_{j}\right|+\sigma_{j}\right)-u_{m_{i}}\right)
$$


After sorting out Equation (22), we can get:

$$
\dot{V}_{i 1} \leq-\frac{1}{2} \widetilde{x}_{i}^{T} Q_{i} \widetilde{x}_{i}-e_{i} \phi_{s_{i}}\left(e_{i}\right)^{T} \hat{\theta}_{s_{i}}-e_{i} u_{m_{i}}+\left|e_{i}\right| \sum_{a=1}^{b_{i}} \Omega\left(t-T_{i a}\right)\left|h_{i a}\left(x, u_{i}\right)\right|
$$

The last item on the right of the Equation (22) can be obtained by using (14):

$$
\left|e_{i}\right| \sum_{a=1}^{b_{i}} \Omega\left(t-T_{i a}\right)\left|h_{i a}\left(x, u_{i}\right)\right| \leq\left|e_{i}\right| \sum_{j=1}^{n} \gamma_{i j}\left(\left|e_{j}\right|\right)
$$

Since $\gamma_{i j}$ is an analytic function, its derivative exists to any degree needed. Therefore, using Taylor's theorem, there is a smooth function $\xi_{i j}$ such that $\gamma_{i j}\left(\left|e_{j}\right|\right)=\gamma_{i j 0}+\left|e_{j}\right| \xi_{i j}\left(\left|e_{j}\right|\right)$, where $\gamma_{i j 0}=\gamma_{i j}(0)$ is a constant. Therefore, by defining $\gamma_{i 0}=\sum_{j=1}^{n} \gamma_{i j}(0)$ and using the inequality $2 \alpha \beta<\alpha^{2}+\beta^{2}, \alpha, \beta \in \Re$, we can get:

$$
\begin{aligned}
\dot{V}_{i 1} & \leq-\frac{1}{2} \widetilde{x}_{i}^{T} Q_{i} \widetilde{x}_{i}-e_{i} \phi_{s_{i}}\left(e_{i}\right)^{T} \hat{\theta}_{s_{i}}-e_{i} u_{m_{i}}+\gamma_{i 0}\left|e_{i}\right|+\left|e_{i}\right| \sum_{j=1}^{n}\left|e_{j}\right| \xi_{i j}\left(\left|e_{j}\right|\right) \\
& \leq-\frac{1}{2} \widetilde{x}_{i}^{T} Q_{i} \widetilde{x}_{i}-e_{i} \phi_{s_{i}}\left(e_{i}\right)^{T} \hat{\theta}_{s_{i}}-e_{i} u_{m_{i}}+\gamma_{i 0}\left|e_{i}\right|+\frac{1}{2} n e_{i}^{2}+\frac{1}{2} \sum_{j=1}^{n} e_{j}^{2} \tilde{\xi}_{i j}^{2}\left(\left|e_{j}\right|\right)
\end{aligned}
$$

Let

$$
s_{i}\left(e_{i}\right)=\gamma_{i 0} \operatorname{sgn}\left(e_{i}\right)+\frac{1}{2} n e_{i}+\frac{1}{2} \sum_{j=1}^{n} e_{j} \xi_{i j}^{2}\left(\left|e_{j}\right|\right)
$$

Use Equation (15) to get:

$$
\dot{V}_{i 1} \leq-\frac{1}{2} \widetilde{x}_{i}^{T} Q_{i} \widetilde{x}_{i}-e_{i} \phi_{s_{i}}\left(e_{i}\right)^{T} \widetilde{\theta}_{s_{i}}+e_{i} \mu_{s_{i}}-e_{i} u_{m_{i}}
$$

Let the Lyapunov function of the $i$-th subsystem be $V_{i}=V_{i 1}+V_{i 2}$, and the time derivative of $\mathrm{V}$ satisfies:

$$
\dot{V}_{i} \leq-\frac{1}{2} \widetilde{x}_{i}^{T} Q_{i} \widetilde{x}_{i}-e_{i} \phi_{s_{i}}\left(e_{i}\right)^{T} \widetilde{\theta}_{s_{i}}+e_{i} \mu_{s_{i}}-e_{i} u_{m_{i}}+\widetilde{\theta}_{s_{i}}^{T} \Gamma_{s_{i}}^{-1} \dot{\hat{\theta}}_{s_{i}}+\frac{1}{\gamma_{s_{i}}}\left(\hat{\mu}_{s_{i}}-\bar{\mu}_{s_{i}}\right) \dot{\hat{\mu}}_{s_{i}}
$$

When $\widetilde{x}_{i}^{T} P_{i} \widetilde{x}_{i}>\lambda_{P_{i}} \varsigma_{i}^{2}$, the value of $u_{m i}$ can be obtained through Equation (17), which can be obtained:

$$
\dot{V}_{i} \leq-\frac{1}{2} \widetilde{x}_{i}^{T} Q_{i} \widetilde{x}_{i}+\widetilde{\theta}_{s_{i}}^{T} \Gamma_{s_{i}}^{-1}\left(\dot{\hat{\theta}}_{s_{i}}-\Gamma_{s_{i}} \phi_{s_{i}}\left(e_{i}\right) e_{i}\right)+\hat{\mu}_{s_{i}}\left(\frac{1}{\gamma_{s_{i}}} \dot{\hat{\mu}}_{s_{i}}-\left|e_{i}\right|\right)+e_{i} \mu_{s_{i}}-\frac{1}{\gamma_{s_{i}}} \bar{\mu}_{s_{i}} \dot{\hat{\mu}}_{s_{i}}
$$

Through (18) and (19), the adaptive rate can be obtained:

$$
\dot{V} \leq-\frac{1}{2} \widetilde{x}_{i}^{T} Q_{i} \widetilde{x}_{i}+e_{i} \mu_{s_{i}}-\left|e_{i}\right| \bar{\mu}_{s_{i}} \leq-\frac{1}{2} \sum_{i=1}^{n} \widetilde{x}_{i}^{T} Q_{i} \widetilde{x}_{i}
$$

Equation (24) indicates that the tracking error $\widetilde{x}_{i}(t)$ will belong to the set $Z_{i}=$ $\left\{\widetilde{x}_{i} \in \Re^{\rho_{i}} \mid \widetilde{x}_{i}^{T} P_{i} \widetilde{x}_{i} \leq \lambda_{P_{i}} \varsigma_{i}^{2}\right\}$ and (20) shows that the time for each subsystem to enter the dead zone is finite, that is, for all $t>0$, there exists $t_{0}<\infty$ such that $\tilde{x}_{i} \in Z_{i}$. The results show the $\left\|\widetilde{x}_{i}\right\|$ and $\zeta_{i}$ uniform ultimate boundedness, and avoid the presence of residual error of approximation parameter drift.

\section{Simulation Process}

In practical applications, due to the large scale of the interconnected system, it is difficult to accurately model the actual system. Therefore, most of the literature for the study of interconnected systems is verified by numerical simulation. Therefore, the rep- 
resentative inverted pendulum [30] was selected as the simulation model in this paper, which is connected by springs to verify the decentralized fault detection and fault-tolerant control method here proposed. We can see the inverted pendulum model in Figure 3. The subsystem model is:

$$
\begin{aligned}
& \dot{x}_{i 1}=x_{i 2} \\
& \dot{x}_{i 2}=\left(\frac{m_{i} g r}{J_{i}}-\frac{k r^{2}}{4 I_{i}}\right) \sin \left(x_{i 1}\right)+\frac{k r}{2 J_{i}}(l-b)+\frac{1}{J_{i}} u_{i}+\sum_{j=1}^{m} \varphi_{i j}\left(x_{j}\right)+\sum_{a=1}^{b_{i}} \Omega\left(t-T_{i}\right) h_{i a}\left(x, u_{i}\right)
\end{aligned}
$$

where $x_{i}(i=1,2)$ represents the state vector, $m_{1}=5 \mathrm{~kg}, m_{2}=6.5 \mathrm{~kg}$ represents the weight of the two pendulums, $J_{1}=0.4 \mathrm{~kg}, J_{2}=0.9 \mathrm{~kg}$ represents the moment of inertia of the two inverted pendulums, $k=105 \mathrm{~N} / \mathrm{m}$ represents the elastic coefficient of the spring, $r=0.8 \mathrm{~m}$ represents the pendulum height, $l=0.6 \mathrm{~m}$ represents the natural length of the spring, and $b=0.5 \mathrm{~m}$ represents the weight of the pendulum. $g=9.81 \mathrm{~m} / \mathrm{s}^{2}$ represents the acceleration of gravity, the interconnection function $\varphi_{12}\left(x_{2}\right)=\left(k r^{2} / 4 J_{1}\right) \sin \left(x_{21}\right) \cos \left(x_{22}\right)$, $\varphi_{21}\left(x_{1}\right)=\left(k r^{2} / 4 J_{2}\right) \sin \left(x_{11}\right)$, let $Q_{i}=I_{2 \times 2}$, the matrix $P_{i}$ satisfy the Lyapunov equation, then

$$
P_{i}=\left[\begin{array}{cc}
1.5 & 0.5 \\
0.5 & 1
\end{array}\right]
$$

where $K_{1}=K_{2}=\left[\begin{array}{ll}1 & 1\end{array}\right]^{T}$, let the threshold value $d_{1}=d_{2}=0.5$. Assuming subsystem 1 external disturbances $h_{1}=k\left(x_{21} x_{12}+0.8\right) \cos \left(x_{21} x_{22}\right)$ occurred in $T_{1}=6 \mathrm{~s}$, subsystem 2 external disturbance $h_{2}=3 k\left(x_{21} x_{12}+1.5 x_{11}\right) \cos \left(x_{11} x_{12}\right)$ occurred in $T_{2}=8 \mathrm{~s}$.

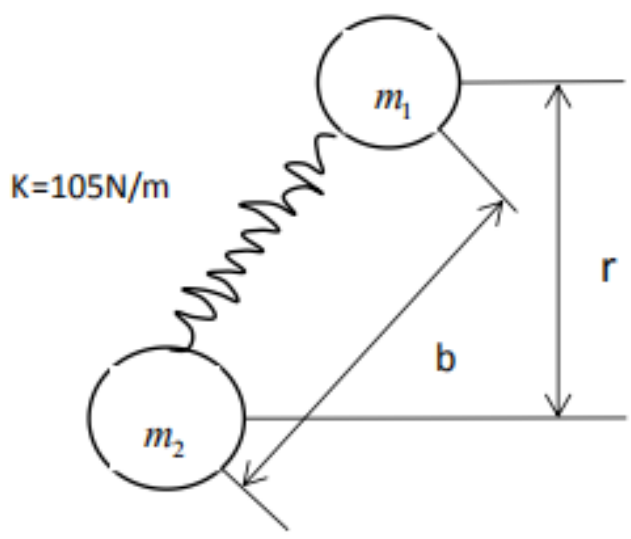

Figure 3. The inverted pendulum model.

Figures 4 and 5 show the fault detection caused by interconnection within subsystem 1 and subsystem 2 by the decentralized fault detection scheme, and compare the different detection thresholds $d_{i}$. It is assumed that subsystem 1 and subsystem 2 cause interconnection failure at $t=2 \mathrm{~s}$ and $t=3 \mathrm{~s}$, respectively, due to the influence of modeling uncertainties. 


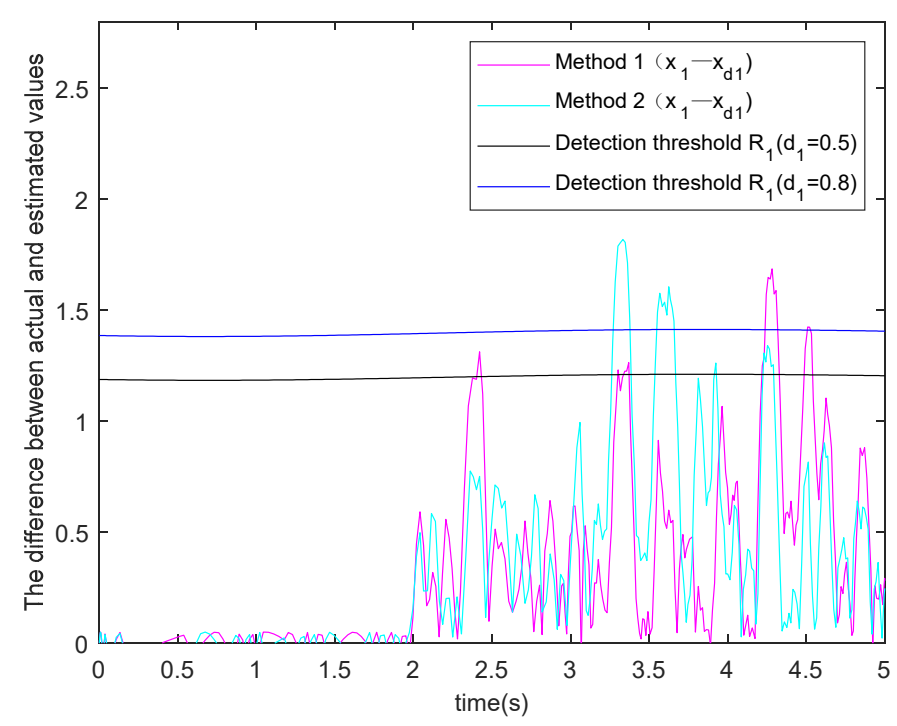

Figure 4. Interconnection fault detection of subsystem 1.

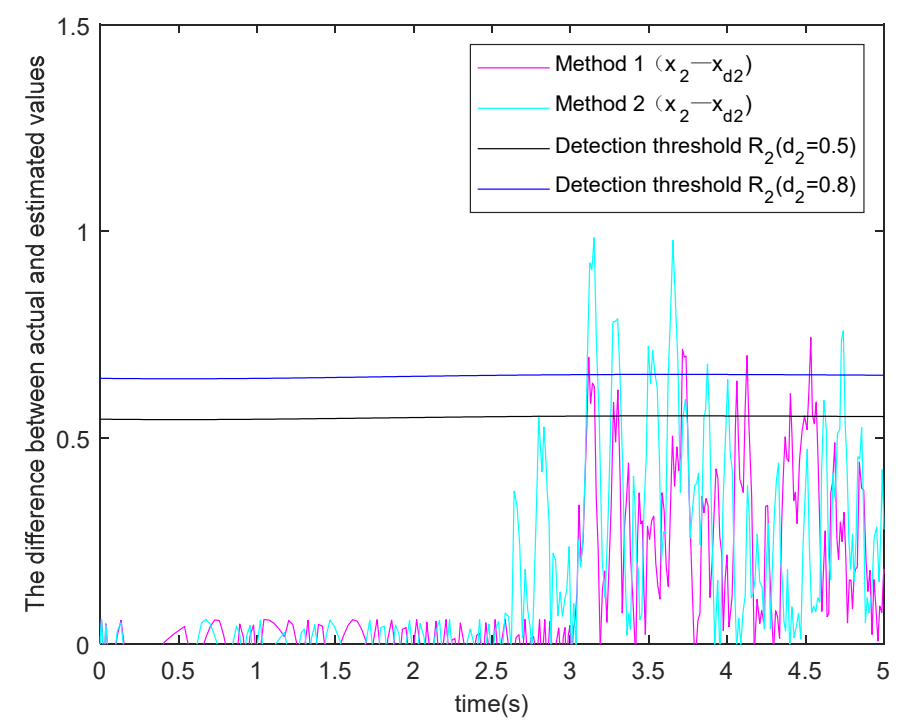

Figure 5. Interconnection fault detection of subsystem 2.

As shown in Figures 4 and 5, Method 1 is the decentralized fault detection scheme designed in this paper based on the communication protocol between subsystems, and Method 2 is the traditional fault detection scheme. After the interconnection failure of subsystem 1, before the interconnection failure of subsystem 2: for Method 1, due to the communication protocol between the subsystems, it can be seen from Figure 5 that the interconnection failure of subsystem 1 has almost no effect on subsystem 2 in the early stage. Because the influence of the two is mutual, so subsystem 2 has less influence on subsystem 1 , and the fault is detected in time at $t=2.4 \mathrm{~s}$. Because there is no communication protocol between the subsystems in Method 2, they are directly connected through the interconnection function, so subsystem 1 directly affects the state vector of subsystem 2 , causing the system state of subsystem 2 to change before interconnection failure occurs, and then the two interact with each other. As a result, the estimation error of subsystem 1 is too small. Compared with the Method 1 designed in this paper, the fault cannot be detected in time, and the system will not alarm.

When an interconnection failure occurs in subsystem 2: in the Method 1, due to the existence of the communication protocol, there is less communication between the subsystems, which reduces the communication burden of the system, so the mutual influ- 
ence between the subsystems is small, the state vector of each subsystem will not change suddenly, and it will not cause irreversible changes to the system. and the fault can be detected in time due to the existence of the detection threshold. However, Method 2 is directly connected through the interconnection function, and the communication between the subsystems is relatively frequent, which affects one another, and increases the communication burden of the system, resulting in large system estimation errors and false alarms. Therefore, compared with Method 2, Method 1 designed in this paper reduces the false alarm and missing alarm rate. In addition, due to the different selection of the communication threshold $d_{i}$, the fault detection threshold $R_{i}$ is also different. It can be seen that as $d_{i}$ increases, the value of $R_{i}$ also becomes larger, that is, the sensitivity of the fault detection scheme to faults decreases. Generally speaking, in Method 1 designed in this paper, by selecting the appropriate value of $d_{i}$, the system can quickly detect the failure caused by the internal interconnection of the subsystem. Due to the existence of the detection threshold, no false alarm will occur in the subsystem.

Figures 6 and 7 show that when a fault is caused by the interconnection in the subsystem, the fault-tolerant control method is used to adjust the system to compensate for the adverse impact caused by the fault.

As shown in the Figures 6 and 7, Method 1 is the decentralized fault-tolerant control method designed in this paper, which implements fault-tolerant control through adaptive adjustment of $u_{i 1}$. Method 2 is to adjust the fault-tolerant control rate by approximating the sum of interconnection faults and external disturbance functions to carry out faulttolerant control for the subsystems. When the fault is caused by the interconnection of the subsystem, it can be found by comparison that the fault-tolerant control method of Method 1 responds quickly, approaches the upper limit of the fault, adaptively adjusts the control rate $u_{i 1}$, and makes the system state quickly restore to stability. Compared with Method 2, the time is shorter.

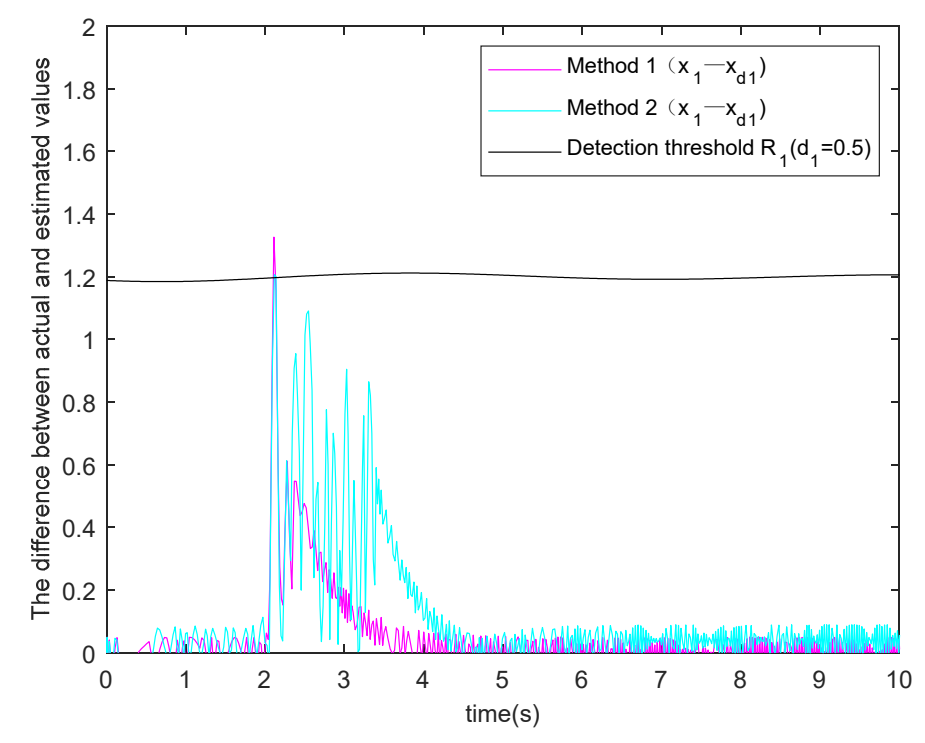

Figure 6. Fault tolerant control of the interconnection fault of subsystem 1 . 


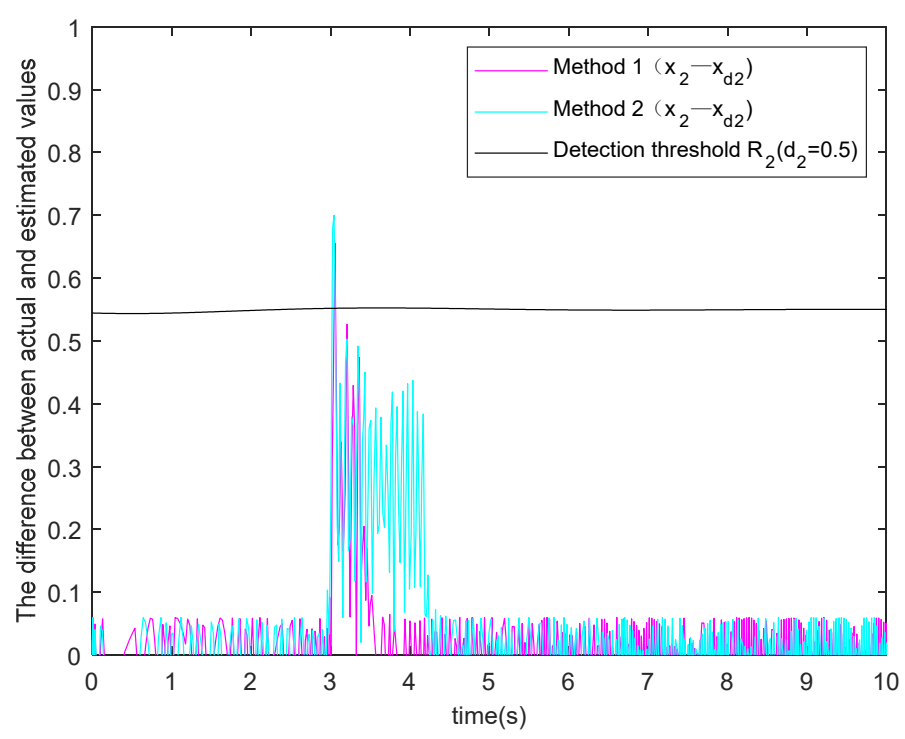

Figure 7. Fault tolerant control of the interconnection fault of subsystem 2.

Figures 8 and 9 show the control effect of the fault-tolerant control method on the system when the subsystem has both internal faults caused by interconnection and external disturbance.

In Figures 8 and 9, Method 1 is the decentralized fault-tolerant control scheme designed in this paper, and Method 2 is to adjust the fault-tolerant control rate to perform fault-tolerant control of the subsystem by approximating the sum of interconnection faults and external disturbance functions. When both types of failure occur, the subsystem can quickly detect the failure due to the existence of the detection threshold. Method 1 is to adaptively approach the upper limit of the interconnection fault and the external disturbance function, and adjust the size of the fault-tolerant control rates $u_{i 1}$ and $u_{i 2}$ to compensate for the adverse effects of the two types of failure respectively. Method 2 adjusts the size of $u_{i 2}$ by approximating the sum of the interconnection faults and external disturbance functions to realize fault-tolerant control of the subsystem. Through comparison, it can be found that the Method 1 designed in this paper makes the system quickly restore stability and consume less time by controlling the two kinds of faults separately.

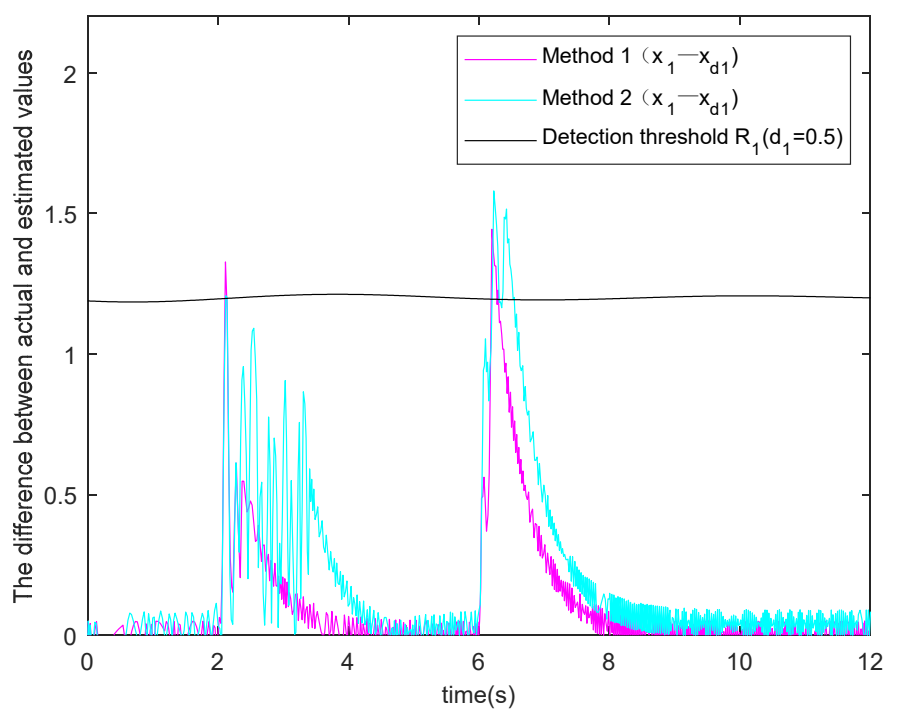

Figure 8. The fault-tolerant control function of subsystem 1 under two fault conditions. 


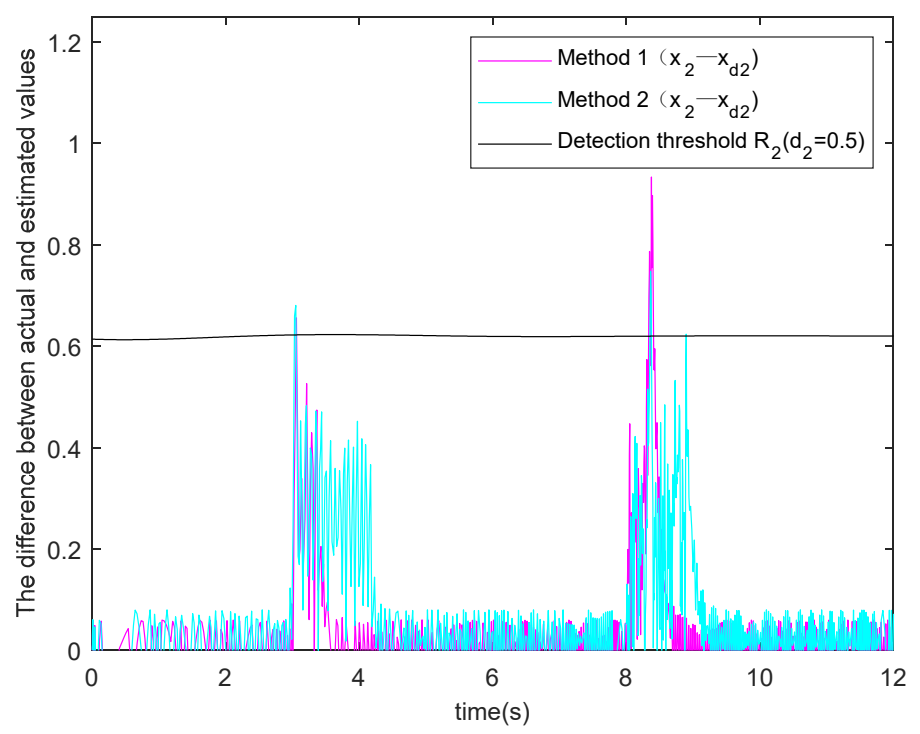

Figure 9. The fault-tolerant control function of subsystem 2 under two fault conditions.

\section{Conclusions}

For nonlinear interconnected systems, this paper proposes a decentralized fault detection and fault-tolerant control scheme. The interconnected system is divided into several subsystems, and the subsystems are connected to each other through unknown interconnection functions. By establishing the communication protocol between the subsystems, the communication between the subsystems is reduced. Only if the norm of the difference between the state vector and the expected vector of the subsystem is greater than the set threshold, will the subsystem pass its own state value to other subsystems, otherwise the interconnection function of other subsystems will use the expected state vector of the current subsystem. It is necessary to design a nonlinear estimator for each subsystem, and to design a fault detection scheme based on this estimator to ensure that faults will not be missed or falsely alarmed. Faults may be caused by the interconnection between subsystems and external disturbances, and multiple disturbances may occur at the same time. Therefore, this paper designs a decentralized fault-tolerant control method based on the adaptive approximation model, which adaptively approaches the upper limit of the external disturbance function, and then based on Lyapunov's theorem, a stable adaptive control rate is obtained, and the influence of the unknown interconnection function and external disturbance is compensated by this control rate. Finally, the simulation results show that the fault can be quickly detected when the fault occurs, and the fault-tolerant control method ensures that the system can quickly recover to a stable state.

This paper also has some shortcomings, because the interconnected system studied is continuous, but in practical application, the interconnected system is not necessarily continuous and may be discrete-this paper assumes that both the interconnection function and the external disturbance are bounded. However, in practical application, both of them are not necessarily bounded, so the method studied in this paper is only valid under specific conditions. If the actual system does not meet these conditions, the system may not operate normally, which is a deficiency of this paper and becomes the target of our next research.

Author Contributions: Investigation, S.Z. and F.W.; writing original draft, S.Z. and J.B.; methodology, S.Z. and F.W. All authors have read and agreed to the published version of the manuscript.

Funding: This research received no external funding.

Institutional Review Board Statement: Not applicable.

Informed Consent Statement: Not applicable. 
Data Availability Statement: The data presented in this study are available on request from the corresponding author.

Conflicts of Interest: The authors declare no conflict of interest.

\section{References}

1. Miljkovic, D. Fault detection methods: A literature survey. In Proceedings of the 34th international convention MIPRO, Opatija, Croatia, 23-27 May 2011; pp. 750-755.

2. Zheng, H.L.; Wang, R.X.; Yang, Y.T. Empirical Analysis of Generalization Performance of Data-driven Fault Diagnosis Method. Chin. J. Mech. Eng. 2020, 56, 116-131.

3. Zhao, J.; OuYang, D.T.; Wang, X.Y. Model-based diagnosis of controller fault in hybrid system. J. Jilin Univ. 2011, 186, 403-407.

4. Yang, F.; Li, X.; Bai, J.J. Nonlinear process quality prediction using wavelet denoising OSC-SVM-PLS. Ind. Eng. Chem. Res. 2020, 59, 6021-6032. [CrossRef]

5. Zhang, S.; Lang, Z.Q. SCADA-data-based wind turbine fault detection: A dynamic model sensor method. Control Eng. Pract. 2020, 102, 104546. [CrossRef]

6. Zuo, L.F.; Yao, L.N. Unknown Input Observer Based Fault Diagnosis and Model Reference Fault Tolerant Control for Quadrotor UAVs. J. Nanjing Univ. Aeronaut. Astronaut. 2020, 6, 889-896.

7. De Souza, R.P.; Marcos, V.M.; Jean, J.L. Fault detection of Discrete-Event Systems based on an identified timed model. Control Eng. Pract. 2020, 105, 104638. [CrossRef]

8. Chen, Z.Q.; Han, L.; Hou, Y.D. Fault Detection and Estimation for a Class of Nonlinear Systems Based on Adaptive Iterative Learning Algorithm. Control Theory Appl. 2020, 37, 144-153.

9. Yao, L.; Zhang, Y. Quality-related Fault Detection for Industrial Processes Based on Adaptive Mixed Kernel Canonical. Control Decis. 2021, 36, 801-807.

10. Zhao, J.; Zhu, R.J.; Chen, P. Fault Detection of Gas Turbine Speed Sensor Based on Fuzzy State Observer. Chin. J. Sens. Actuators 2019, 32, 1227-1231.

11. Tao, L.Q.; Liu, C.; Wang, W. Fault Detection in Aircraft Engines Based on Adaptive Sliding Mode Observer. Comput. Simul. 2019, $36,67-73$.

12. Wang, N.; Li, H.; Wu, F. Fault Diagnosis of Complex Chemical Processes Using Feature Fusion of a Convolutional Network. Ind. Eng. Chem. Res. 2021, 60, 2232-2248. [CrossRef]

13. Huang, R.R.; Li, Y.G.; Hou, Y.D. Intelligent Vehicle Fault Detection and Isolation based on spatial Geometry Method. Syst. Simul. Technol. 2017, 3, 28-33.

14. Yu, W.; Jiang, D.; Wang, J. Rotor-current-based fault detection for doubly-fed induction generator using new sliding mode observer. Trans. Inst. Meas. Control 2020, 42, 3110-3122. [CrossRef]

15. Wang, N.; Yang, F.; Zhang, R.; Gao, F. Intelligent Fault Diagnosis for Chemical Processes Using Deep Learning Multimodel Fusion. IEEE Trans. Cybern. 2020, 99, 1-15.

16. Chen, Z.Q.; Han, L.; Hou, Y.D. Fault detection and estimation based on adaptive iterative learning algorithm for nonlinear systems. Control Theory Appl. 2020, 37, 837-846.

17. Straka, O.; Čochář, I.P. Decentralized and distributed active fault diagnosis: Multiple model estimation algorithms. Int. J. Appl. Math. Comput. Sci. 2020, 30, 239-249.

18. Zhang, X.; Polycarpou, M.M.; Parisini, T. Decentralized Fault Detection in a Class of Large-Scale Nonlinear Uncertain Systems. In Proceedings of the 2010 American Control Conference, Baltimore, MD, USA, 30 June-2 July 2010; pp. 6988-6993.

19. Stanković, S.S.; Ilić, N.; Djurović, Ž.; Stanković, M.; Johansson, K.H. Consensus based overlapping decentralized fault detection and isolation. In Proceedings of the 2010 Conference on Control and Fault-Tolerant Systems (SysTol), Nice, France, 6-8 October 2010; pp. 570-575.

20. Zhang, R.D.; Lu, R.Q.; Xue, A.K.; Gao, F.R. New minmax linear quadratic fault-tolerant tracking control for batch processes. IEEE Trans. Autom. Control 2016, 61, 3045-3051. [CrossRef]

21. Luo, W.P.; Wang, L.M.; Zhang, R.D.; Gao, F. 2D Switched Model-Based Infinite Horizon LQ Fault-Tolerant Tracking Control for Batch Process. Ind. Eng. Chem. Res. 2019, 58, 9540-9551. [CrossRef]

22. Qin, Z.H.; He, X.X.; Li, G. Adaptive Decentralized Tracking Control for Nonlinear Interconnected Systems with Input Quantization and Output Constraints. Acta Autom. Sin. 2019, 8, 1250-1258.

23. Shi, H.T.; Chen, B.; Ge, H.L.; Liu, Z. Adaptive Neural Network Decentralized Control for Non-strict Feedback Large-scale Systems. J. Qingdao Univ. 2016, 369, 748-764.

24. Fu, Q. Decentralized iterative learning control for large interconnected nonlinear distributed parameter systems. J. Syst. Sci. Math. Sci. 2016, 36, 1557-1573.

25. Tong, S.C.; Li, Y.M.; Zhang, H.G. Adaptive Neural Network Decentralized Backstepping Output—Feedback Control for Nonlinear Large-Scale systems with Time Delays. IEEE Trans. Neural Netw. 2011, 22, 1073-1086. [CrossRef] [PubMed]

26. Wang, H.Q.; Chen, B.; Lin, C. Adaptive Fuzzy Decentralized Control for a Class of Large-Scale Stochastic Nonlinear Systems. Neurocomputing 2013, 103, 155-163. [CrossRef]

27. Ferdowsi, H.; Jagannathan, S. A decentralized fault accommodation scheme for nonlinear interconnected systems. In Proceedings of the 2013 IEEE Conference on Prognostics and Health Management (PHM), Gaithersburg, MD, USA, 24-27 June 2013. 
28. Panagi, P.; Polycarpou, M.M. Distributed fault detection and accommodation with partial communication. In Proceedings of the 2010 4th International Symposium on Communications, Control and Signal Processing (ISCCSP), Limassol, Cyprus, 3-5 March 2010.

29. Panagi, P.; Polycarpou, M.M. Decentralized Fault Tolerant Control of a Class of Interconnected Nonlinear Systems. IEEE Trans. Autom. Control 2011, 56, 178-184. [CrossRef]

30. Guo, T. Adaptive fault-tolerant fuzzy control of interconnected double inverted pendulum. Comput. Eng. Appl. 2017, 53, 261-270. 